\title{
A RARE CASE OF AN OFF MIDLINE THYROGLOSSAL DUCT CYST PAPILLARY THYROID CARCINOMA : Case presentation
}

\author{
Melania Balaş ${ }^{1}$, Ioana Golu${ }^{1}$, Daniela Amzar ${ }^{1}$, Mihaela Vlad $^{1}$, Emilia lanes ${ }^{2}$, Alis Dema ${ }^{3}$, loana Zosin ${ }^{1}$ \\ ${ }^{1}$ Department of Endocrinology, ${ }^{2}$ Department of Maxillofacial Surgery , ${ }^{3}$ Department of Morphopathology \\ University of Medicine and Pharmacy "V. Babeş" Timişoara, Romania
}

Background: Papillary thyroid carcinoma can be found very rarely in a thyroglossal duct cyst (TDC) (1\%), this cancer variant being the most common type. Although most of the TDC develop in the midline of the thyroglossal duct (approximately 70\%), some cysts occur off midline, within $2 \mathrm{~cm}$ of the midline.

Case presentation: The female patient of 34-year-old presented at the Maxillofacial Surgery Department in October 2014, with a right submandibular painless neck mass at about $1.5 \mathrm{~cm}$ of the midline. The lump was discovered by the patient during pregnancy and increased in size gradually, reaching $2.5 \mathrm{~cm}$ at 5 months after birth. The clinical examination did not reveal any pathological aspects of the nose, nasopharynx, or cervical lymph nodes. The ultrasound of the swelling revealed a pseudosolid mass near the hyoid bone, with thick capsule (4 $\mathrm{mm}$ ), microcalcifications and internal vascularity.

The post-contrast computed tomography (CT) showed a $28 / 23 \mathrm{~mm}$ right submandibular mass, with complex heterogeneous composition, thick capsule, several microcalcifications in the solid component, no adenopathies, and no surrounding infiltration (Figure 1).

The evaluation of the thyroid gland revealed: normal appearance of the thyroid gland at the ultrasound, CT (Figure 2), thyroid scintigraphy, euthyroidism (normal TSH, FT4), negative serum antithyroid antibodies, and normal serum thyroglobulin.

Figure 1. Computed tomography showing a $28 / 23 \mathrm{~mm}$ right submandibular mass, with complex composition, thick capsule, several microcalcifications in the solid component

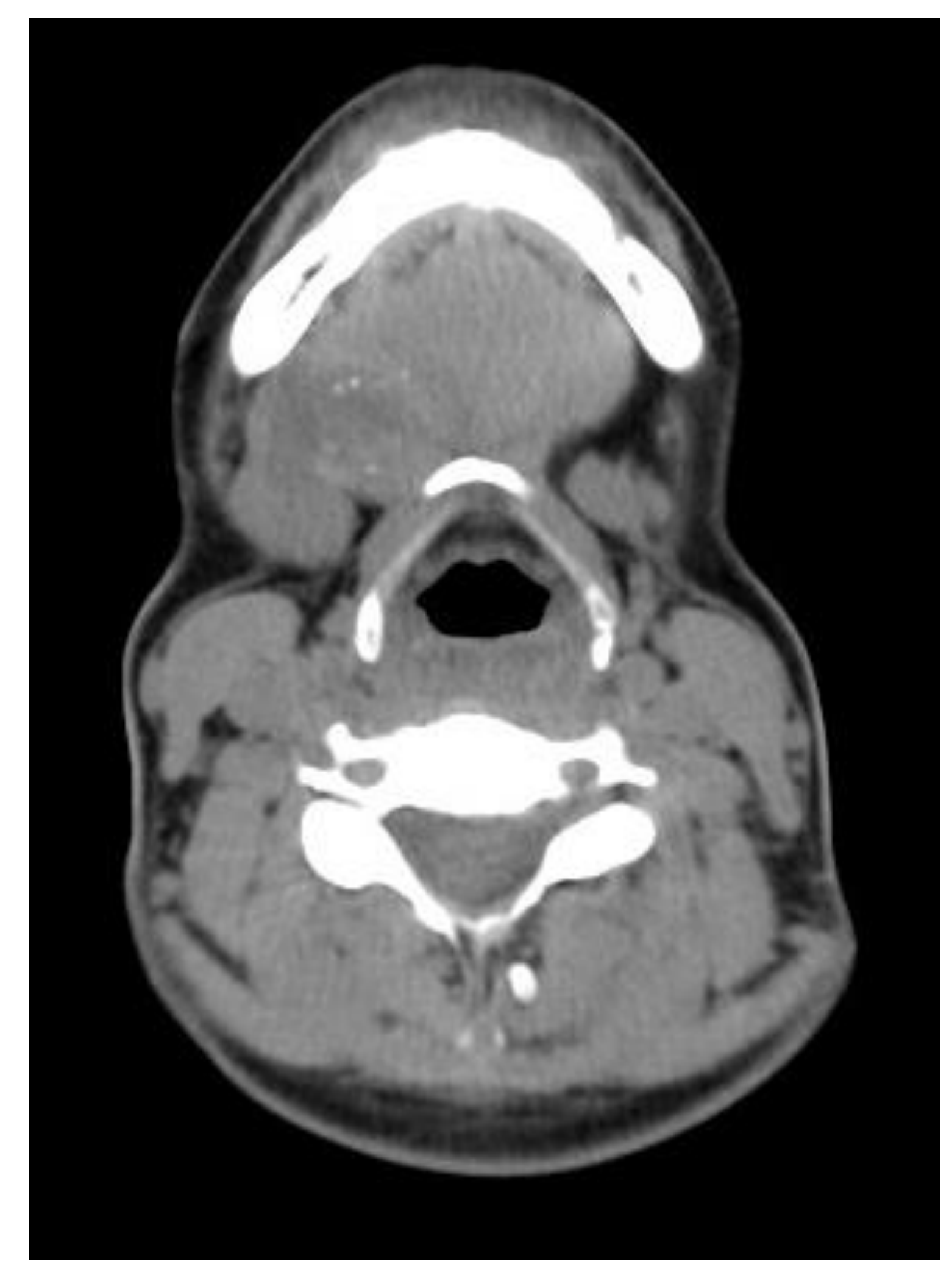

Figure 2. Computed tomography showing normal thyroid gland

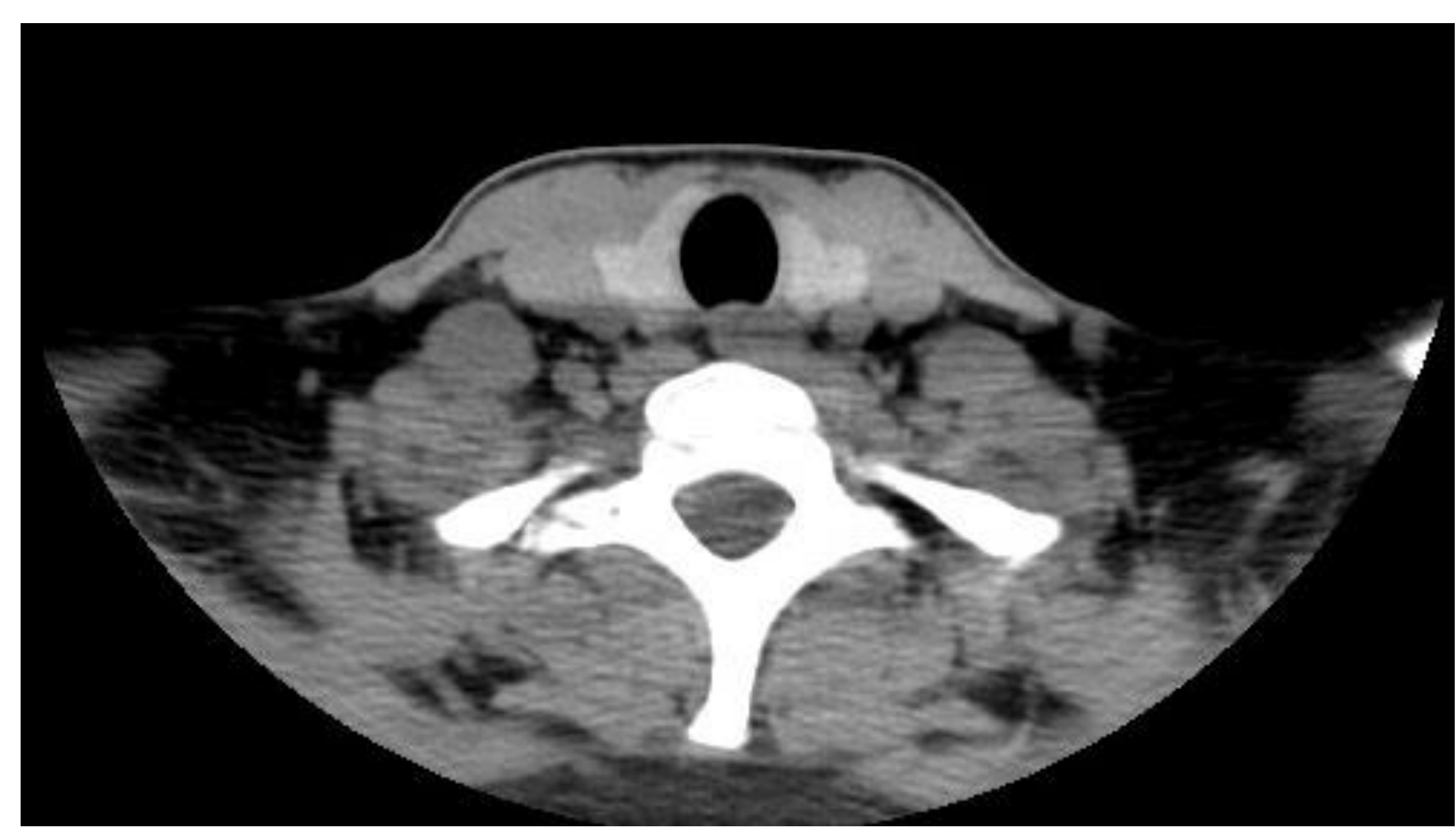

Figure 3. Papillary thyroid carcinoma in TDC (HEx40)

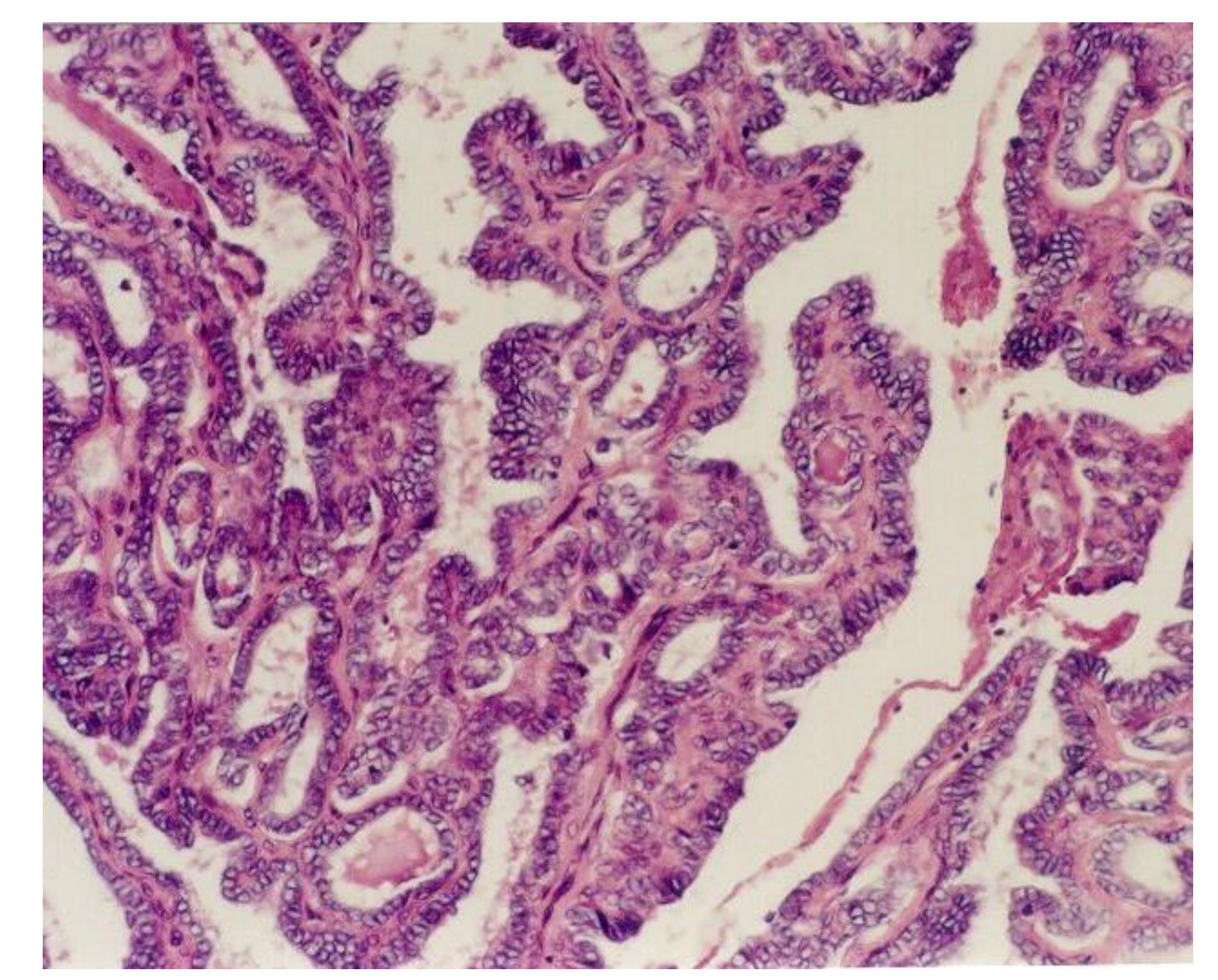

After the mass was removed, histological examination established the diagnosis of papillary thyroid carcinoma (Figure 3).

Follow-up: Up to now, the patient (still breastfeeding) has been closely monitored by clinical examination and thyroid ultrasound. Suppressive thyroxin treatment was commenced.

Discussion and conclusion: The clinical picture of an TDC carcinoma resembles often that of a benign TDC. As TDC in adults presents variable sonographic and CT appearance, the diagnosis poses sometimes difficulties. Moreover, the diagnosis can be hampered by the unusual off line location of the TDC. In the presented case, the final diagnosis was made after surgery. The management of the TDC carcinoma is still controversial, the recommendations ranging from only Sistrunk procedure to total thyroidectomy, followed by radioiodine and suppressive thyroxin treatment, or only suppressive doses of thyroxin.

\section{References:}

1. Park MH, Yoon JH, Jegal YJ, Lee JS. Papillary thyroglossal duct cyst carcinoma with synchronous occult papillary thyroid microcarcinoma. Yonsei Med J. 2010;51(4):609-611.

2. Branstetter BF, Weissman JL,Kennedy TL, Whitake M. The CT appearance of thyroglossal duct carcinoma. Am J Neuroradiol. 2000; 21:1547-1550. 3.Gebbia V, Di Gregorio C, Attard M. Thyroglossal duct cyst carcinoma with concurrent thyroid carcinoma: a case report. J Med Case Rep. $2008 ; 2: 132$. 